\author{
Kouhei Ishiwari · Masaharu Kotani · Minoru Suzuki \\ Elena Pumbo · Akemi Suzuki · Toshihide Kobayashi \\ Tamaki Ueno · Tomoko Fukushige · Tamotsu Kanzaki \\ Masato Imada $\cdot$ Kohji Itoh $\cdot$ Shinji Akioka \\ Youichi Tajima $\cdot$ Hitoshi Sakuraba
}

\title{
Clinical, biochemical, and cytochemical studies on a Japanese Salla disease case associated with a renal disorder
}

Received: 27 July 2004/ Accepted: 6 September 2004/Published online: 13 November 2004

(c) The Japan Society of Human Genetics and Springer-Verlag 2004

\begin{abstract}
We report the first Japanese case of Salla disease. A 5-year-old male patient developed unique proteinuria with other clinical manifestations, including coarse facies, dysostosis multiplex, mild mitral valve regurgitation, umbilical and inguinal herniation, and mild developmental delay. Pathological analysis of biopsied kidney tissues showed marked vacuolation of podocytes, mesangial cells, capillary endothelial cells, and tubular cells. Biochemical studies involving thinlayer chromatography and mass spectrometry revealed
\end{abstract}

K. Ishiwari $\cdot \mathrm{T}$. Ueno

Department of Pediatrics,

Ayabe Municipal Hospital, Ayabe, Japan

M. Kotani $\cdot$ Y. Tajima $\cdot$ H. Sakuraba $(\bowtie)$

Department of Clinical Genetics,

The Tokyo Metropolitan Institute of Medical Science,

Tokyo Metropolitan Organization for Medical Research,

3-18-22 Honkomagome, Bunkyo-ku, Tokyo 113-8613, Japan

E-mail: sakuraba@rinshoken.or.jp

Tel.: + 81-3-38232105

Fax: + 81-3-38236008

M. Suzuki $\cdot$ E. Pumbo $\cdot$ A. Suzuki $\cdot$ T. Kobayashi Supra-Biomolecular Research System,

RIKEN Frontier Research System,

RIKEN, Wako, Japan

T. Fukushige $\cdot$ T. Kanzaki

Department of Dermatology,

Kagoshima University Graduate School of Medical

and Dental Sciences, Kagoshima, Japan

M. Imada

Department of Anatomy,

Nihon University School of Medicine, Tokyo, Japan

K. Itoh

Division of Medicinal Biotechnology, Institute of Medical

Resources, Faculty of Pharmaceutical Sciences,

The University of Tokushima, Tokushima, Japan

S. Akioka

Department of Pediatrics,

Kyoto Prefectural University of Medicine,

Kyoto, Japan increased excretion of free sialic acid ( $N$-acetylneuraminic acid) into the patient's urine. Immuno- and lectin staining of the patient's cells demonstrated the accumulation of sialyl and asialyl glycoconjugates in lysosomes and late endosomes. A defect in sialyl glycoconjugate metabolism is thought to have occurred in the patient's cells, besides impairment of the lysosomal transport of free sialic acid residues. A renal disorder should be considered as an important manifestation, not only in infantile free sialic acid storage disease but also in Salla disease.

Keywords Salla disease $\cdot$ Infantile free sialic acid storage disease $\cdot$ Sialic acid $\cdot$ Lectin $\cdot$ Mass spectrometry $\cdot$ Proteinuria

\section{Introduction}

Lysosomal free sialic acid storage disorders are characterized by increased urinary free sialic acid excretion and its accumulation in lysosomes (reviewed by Aula and Gahl 2001). They are caused by impaired anion transport across the lysosomal membrane (Mancini et al. 1991), and the responsible gene has been identified as the SLC17A5-gene (Verheijen et al. 1999; Aula et al. 2000) located on the long arm of chromosome 6.

The disorders include two main clinical phenotypes: infantile free sialic acid storage disease (ISSD) and Salla disease. The former is a rapidly progressive form with fetal or neonatal ascites, psychomotor retardation, coarse facies, hepatosplenomegaly, and hypopigmented skin/hair and leads to death in early infancy. Salla disease is a milder form of the disorder. Patients with Salla disease are usually normal at birth but develop mental retardation and ataxia, and their life span is slightly reduced or normal. Most Salla disease patients originate from Finland and Sweden, with a few cases elsewhere. 
Although disease-causing mutations in the responsible gene and lysosomal accumulation of free sialic acid in various tissues have been reported, the pathophysiology of the disease has not been clarified yet in detail.

As to renal disorders in lysosomal free sialic acid storage diseases, Sperl et al. (1990) reported two Austrian ISSD siblings who developed steroid-resistant congenital nephrosis, which had been thought to be an unusual presentation of ISSD. However, Lemyre et al. (1999) showed that a nephrotic syndrome occurred in four of seven ISSD patients in whom renal evaluation was performed, and ISSD seems to be an important cause of nephrosis in infants with a storage disorder phenotype.

We report here the first Japanese Salla disease patient with a unique renal disorder and describe biochemical and cytochemical investigation of this patient.

\section{Patient and methods}

\section{Case report}

This male patient is the 5-year-old child of healthy nonconsanguineous Japanese parents. The family history showed no abnormality. He was born by means of caesarean section at 38 weeks of gestation because of fetal distress. The Apgar scores were 9 and 10 at 1 and $5 \mathrm{~min}$, respectively. His birth weight was $2,116 \mathrm{~g}$. Moderate psychomotor developmental delay was noticed at 4 months of age. At the age of 11 months, proteinuria and moderate mitral valve regurgitation were found. Magnetic resonance imaging (MRI) analy- ses revealed an enlargement of the third and lateral ventricles, indicating brain atrophy.

He was admitted to our hospital at age 2 years for detailed examinations, including a renal biopsy. On physical examination, he had findings suggestive of a storage disease: coarse facies, partial deformity of the thorax, a systolic heart murmur, and umbilical and inguinal herniation. Hepatosplenomegaly, angiokeratoma, or hypopigmentation of the skin/hair was not found. X-ray examinations revealed signs of dysostosis multiplex. Ophthalmological examinations showed no cherry-red spots or corneal clouding. A peripheral smear was negative for storage cells at that time. A blood chemistry test showed normal findings. Urinalysis revealed proteinuria $(300-600 \mathrm{mg} / \mathrm{dl})$. Urinary mucopolysaccharides were normal, and urinary oligosaccharides showed no diagnostic pattern.

The activities of $\beta$-galactosidase, $\beta$-hexosaminidase, $\beta$-mannosidase, and aspartylglucosaminidase in plasma, and those of $\beta$-galactosidase, $\alpha$ - $N$-acetylgalactosaminidase, $\alpha$-fucosidase, and $\alpha$-mannosidase in cultured fibroblasts were all normal. The total sialic acid concentration in urine was measured using a commercially available kit (Kyokuto Pharmaceutical and Industrial Co., Tokyo, Japan) and was found to be increased in the patient's urine $(788 \mu \mathrm{g} / \mathrm{mg} \mathrm{Cr})$ compared to age-matched control subjects $[152 \pm 25 \mu \mathrm{g} / \mathrm{mg} \mathrm{Cr}(4)$, mean $\pm \mathrm{SD}(n)]$.

Light microscopic analyses of biopsied kidney tissues revealed marked vacuolation of the cytoplasm in podocytes, mesangial cells, capillary endothelial cells, and tubular epithelial cells (Fig. 1a, b). At the ultrastructural level, these cells contained membrane-bound
Fig. 1 Pathological findings in the biopsied renal tissue of the patient. a Light microscopy of the glomerular region. Periodic acid-Schiff staining, $\times 1,000$. b Light microscopy of the proximal tubulus. Periodic acidSchiff staining, $\times 1,000$. c Electron microscopy of the glomerular region. Bar $=5 \mu \mathrm{m}$
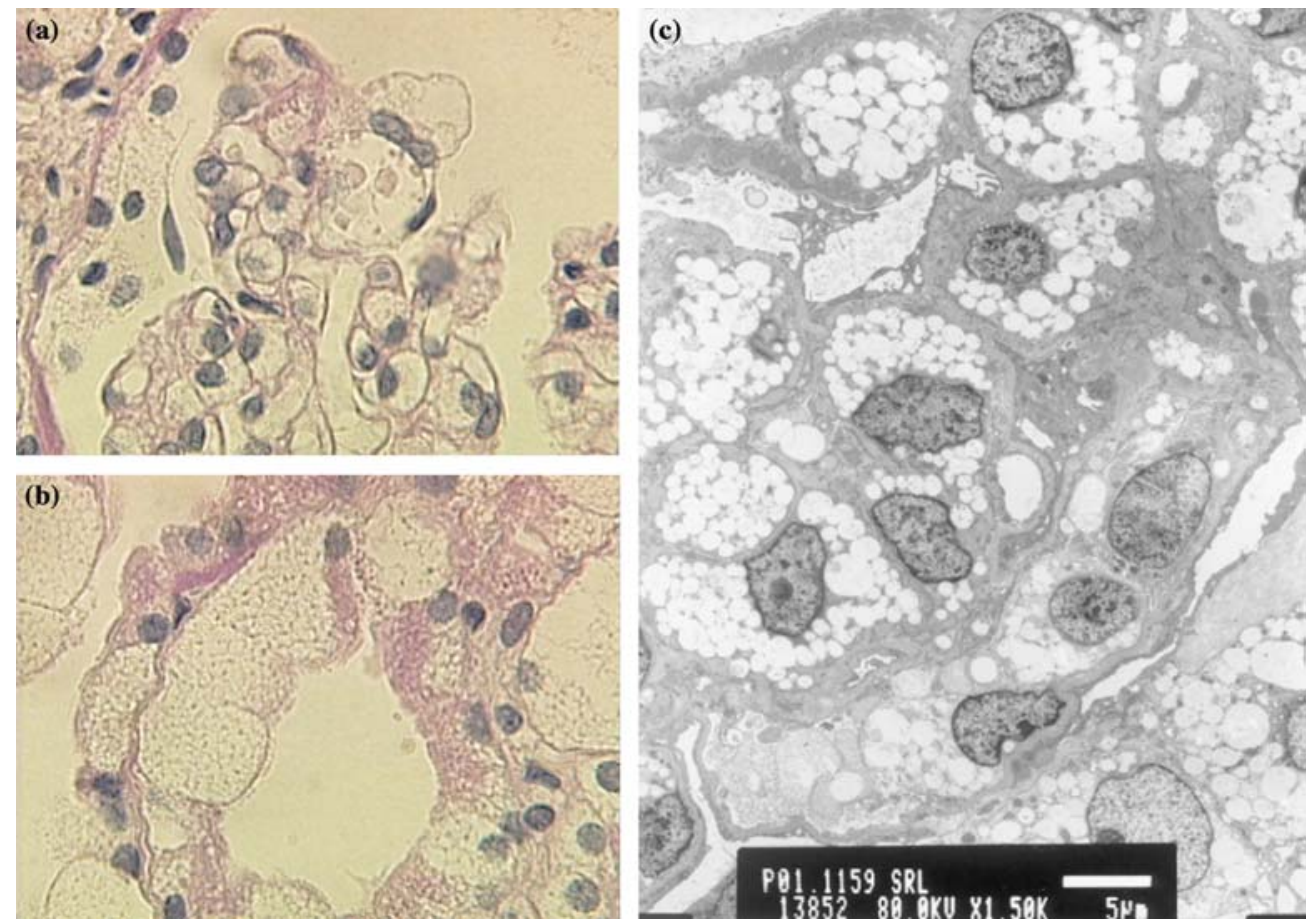
vacuoles, most of which were optically empty but some of which were filled with fine granules (Fig. 1c). The same pathological findings were found for vascular endothelial cells, eccrine sweat gland cells, fibroblasts, and neuronal cells from the biopsied skin tissue.

At the age of 5 years, the patient showed slowly and progressively delayed psychomotor development, and vacuolated lymphocytes were found in a peripheral smear. MRI showed basically the same findings as those at the age of 11 months.

Assaying of the enzymes involved in sialic acid metabolism

Cultured skin fibroblasts from the patient and control subjects were established and maintained in our laboratory. The cells were cultured in Ham's F-10 medium containing $10 \%$ fetal calf serum and antibiotics at $37^{\circ} \mathrm{C}$ in a humidified incubator flushed continuously with a $5 \% \mathrm{CO}_{2}-95 \%$ air mixture.

The activities of lysosomal sialidase and acid carboxypeptidase in cell extracts were assayed, as described previously, using 4-methylumbelliferyl $N$-acetylneuraminic acid and $N$-carbobenzoxy- $L$-phenylalanyl- $L$-leucine as substrates, respectively (Itoh et al. 1997). Protein determination was performed with a DC protein assay kit (Bio-Rad, Hercules, CA, USA) using bovine serum albumin as a standard.

Purification of accumulated glycoconjugates in the patient's urine

Urinary samples from the patient and a control subject were lyophilized, and the dried materials were suspended in water. The insoluble materials were removed by centrifugation at $1,500 \times \mathrm{g}$ for $20 \mathrm{~min}$. The supernatant was adjusted to half the volume of the original urine. The urine sample, $2 \mathrm{ml}$, which is equivalent to $4 \mathrm{ml}$ original urine, was applied to a Bio-Gel P2 column (Bio-Rad, $3 \mathrm{~cm}$ i.d. $\times 56 \mathrm{~cm}$ ), eluted with water, and separated into 3-ml fractions. An aliquot, $1 \mathrm{ml}$, of each fraction was lyophilized and suspended in $30 \mu \mathrm{l}$ of water, and then $5 \mu \mathrm{l}$ was applied to a silica gel thinlayer chromatography plate (HPTLC plate; Merck, Damstadt, Germany).

The plates were developed with the solvent system, ethanol:acetic acid: water, $5: 2: 1 \mathrm{v} / \mathrm{v}$, and then spots were detected by spraying resorcinol or orcinol reagent and heating the plates at 95 and $110^{\circ} \mathrm{C}$, respectively.

The fractions representing the major resorcinol-positive spot for the patient's urine were pooled and lyophilized. The dried materials were dissolved in $2.4 \mathrm{ml}$ of the solvent system, $n$-butanol:ethanol:water, 7:1:1, v/ $\mathrm{v}$, and then applied to a cellulose cartridge (Takara Bio, Kyoto, Japan), which had been prewashed with $10 \mathrm{ml}$ of water, $10 \mathrm{ml}$ of ethanol:water, $1: 1, \mathrm{v} / \mathrm{v}$, and $10 \mathrm{ml}$ of $n$ buthanol:ethanol:water, 7:1:1,v/v. Materials were eluted with $10 \mathrm{ml}$ of $n$-butanol:ethanol:water, 7:1:1, v/v, ethanol:water, 1:1, v/v, and then water. An aliquot of each fraction was analyzed by thin-layer chromatography (TLC) with the solvent system, ethanol:acetic acid:water, 5:2:1, v/v, and resorcinol reagent detection. The resorcinol-positive spot was recovered with ethanol:water, $1: 1, \mathrm{v} / \mathrm{v}$, as a single band.

\section{Mass spectrometry}

The material obtained with the cellulose cartridge with ethanol:water, 1:1, v/v, was analyzed by matrix-assisted laser desorption/ionization time-of-flight (MALDITOF) mass spectrometry using a Kratos Axima CFR (Shimazu Co., Kyoto, Japan) and 2,5-dihydroxybenzoic acid as a matrix.

Determination of the free sialic acid contents in urine samples

The patient's and controls' urine samples were centrifuged at $1,500 \times \mathrm{g}$ for $15 \mathrm{~min}$, and the supernatants, $1 \mathrm{ml}$ each, were mixed with $1 \mathrm{ml}$ of ethanol and $8 \mathrm{ml}$ of $n$-butanol. The mixtures were centrifuged at $1,500 \times \mathrm{g}$ for $15 \mathrm{~min}$, and then the supernatants were applied to cellulose cartridges prepared in the same way as above, except for the use of $n$-butanol:ethanol:water, $8: 1: 1, \mathrm{v} / \mathrm{v}$, instead of $7: 1: 1, \mathrm{v} / \mathrm{v}$. The materials were eluted with $10 \mathrm{ml}$ each of $n$-butanol:ethanol:water, 8:1:1 and 4:1:1, $\mathrm{v} / \mathrm{v}$, and ethanol:water, 1:1, v/v. Free sialic acid was detected in the fraction obtained with $n$-butanol:ethanol:water, $4: 1: 1, \mathrm{v} / \mathrm{v}$, on TLC and subjected to quantitative determination by high-performance liquid chromatography (HPLC) as the 1,2-diamino-4,5methylenedioxybenzene derivative using a reagent kit for fluorescence derivatization of sialic acid (Takara Bio) according the method of Hara et al. (1989).

Immuno- and lectin staining of cultured fibroblasts

For immunostaining of cultured fibroblasts, monoclonal mouse antihuman lysosome-associated membrane protein-1 (Lamp-1; Southern Biotechnology, Birmingham, AL, USA), monoclonal antihuman lysosome-associated membrane protein-2 (Lamp-2; Southern Biotechnology), monoclonal mouse anti-CD63 (Immunotechs, Marseille, Codex, France), monoclonal mouse antilysobisphosphatidic acid (LBPA; Kobayashi et al. 1999), and anti-Tn antigen (DAKO, Glostrup, Denmark) were used as the first antibodies. For lectin staining of cultured fibroblasts, biotin-conjugated lectins Maackia amurensis (MAM; Seikagaku Co., Tokyo, Japan), Jacalin (Seikagaku Co.), Arachis hypoogaea (PNA; Seikagaku Co.), Datura stramonium (DSA; Seikagaku Co.), Phaseolus vulgaris (PHA; Seikagaku Co.), and wheat germ agglutinin (WGA, Seikagaku Co.) were used. 
Cy3-conjugated goat antimouse IgG $\mathrm{F}\left(\mathrm{ab}^{\prime}\right)_{2}$ (Jackson Immuno Research, West Grove, PA, USA) and streptoavidin-FITC (SAv-FITC; BD Bioscience, San Jose, CA, USA) were used for the second immunoreaction and biotin/streptoavidin reaction, respectively.

Cultured fibroblasts from the patient and normal control subjects (approximately $4 \times 10^{3}$ cells) were cultured on Lab-Tek chamber slides (Nunk, Naperville, IL, USA) for 3 days. The cells were then fixed with $4 \%$ paraformaldehyde in phosphate-buffered saline (PBS; $\mathrm{pH}$ 7.4) for 5 min. After the fixing solution had been removed, the cells were washed with PBS and then pretreated with $1 \%$ bovine serum albumin (BSA) in PBS for $30 \mathrm{~min}$. Then, the cells were reacted with one of the first antibodies (1:100 diluted) or one of the lectins $(10 \mu \mathrm{g} / \mathrm{ml})$ for $1 \mathrm{~h}$. After washing five times with $1 \%$ BSA-PBS, the cells were treated with a second immunoreaction substrate (Cy3-conjugated goat antimouse IgG $\mathrm{F}\left(\mathrm{ab}^{\prime}\right)_{2}, 1: 200$ diluted; and SAv-FITC, 1:100 diluted) for $1 \mathrm{~h}$ in the dark. The stained cells were examined under a microscope (Axiovert $100 \mathrm{M}$; Carl Zeiss,
Oberkochen, Germany) equipped with a confocal laser scanning imaging system (LSM 510; Carl Zeiss).

Lectin blotting of cultured fibroblast lysates was performed according to the method of Kotani et al. (2004). Cell lysates were separated by sodium dodecyl sulfate-polyacrylamide gel electrophoresis under nonreducing conditions in 5-20\% gradient gels and then electroblotted onto polyvinylidene diflouride membranes (Immobilon; Nippon Millipore, Tokyo, Japan). Then, the membranes were incubated with biotinylated MAM $(1.0-5.0 \mathrm{ng} / \mathrm{ml})$ for $1 \mathrm{~h}$ followed by treatment with peroxidase-conjugated mouse antibiotin (1:200 diluted; ZYMED, San Francisco, CA, USA). The MAMreacting bands were visualized with an ECL detection system (Amersham Pharmacia Biotech, Buckinghamshire, UK). Proteins on the blots were stained with Coomassie brilliant blue (Merck). In this study, cultured fibroblasts from a patient with ISSD and patients with sialidosis, in which sialyl oligosaccharides and glycoproteins accumulate, were used as pathological controls, as well as ones from the patient and a normal subject.
Fig. 2 Bio-Gel P2 column chromatography of the patient's and controls' urine. Concentrated urine $(2 \mathrm{ml})$ equivalent to $4 \mathrm{ml}$ of the original urine sample was applied to a $\mathrm{P} 2$ column, and the effluent was separated into 3-ml fractions. A one-sixth aliquot was applied on a thin-layer chromatography (TLC) plate and detected with resorcinol reagent. In the patient's urine, the major resorcinol-positive band was eluted as fraction numbers $48-53$
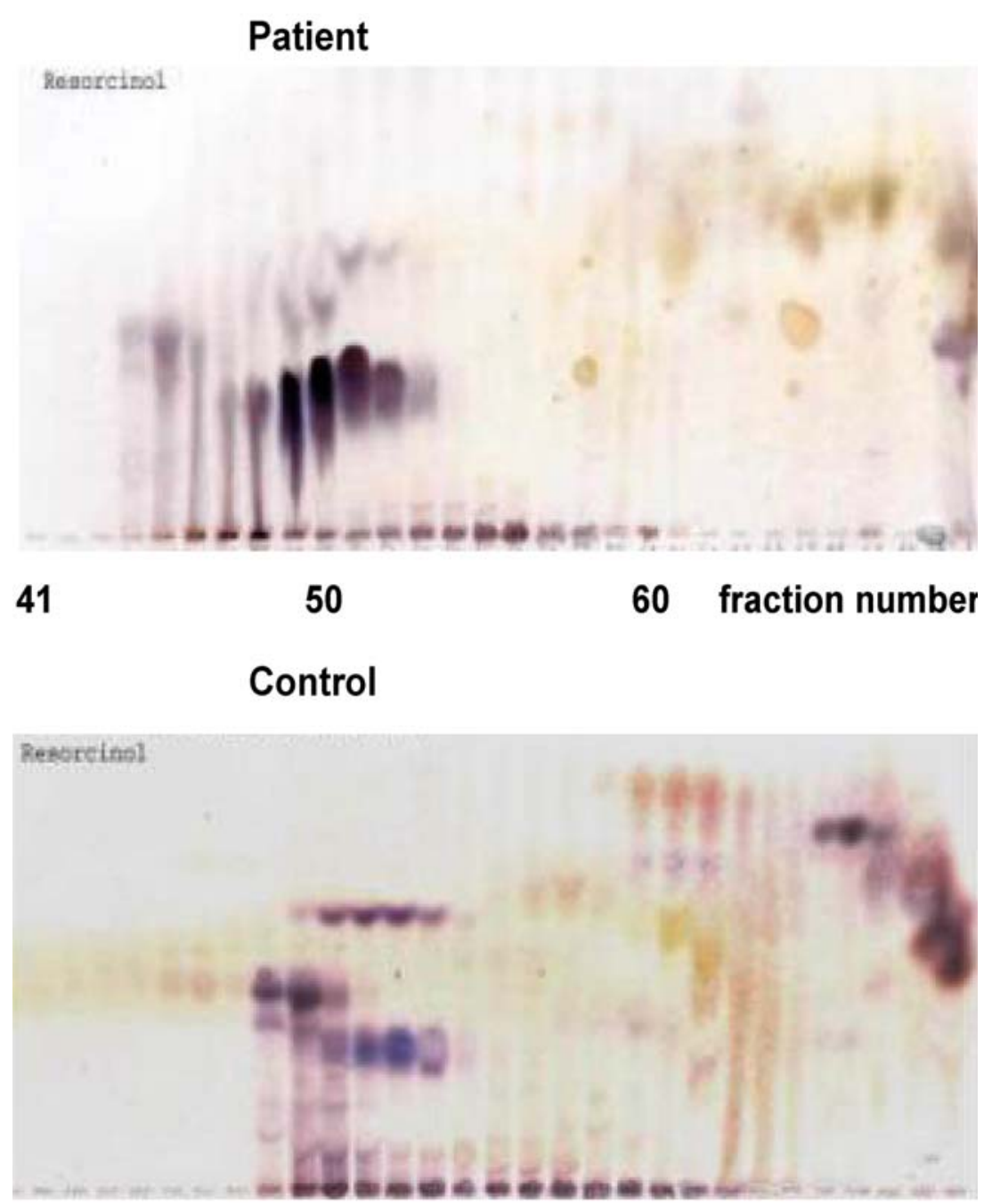
orcinol

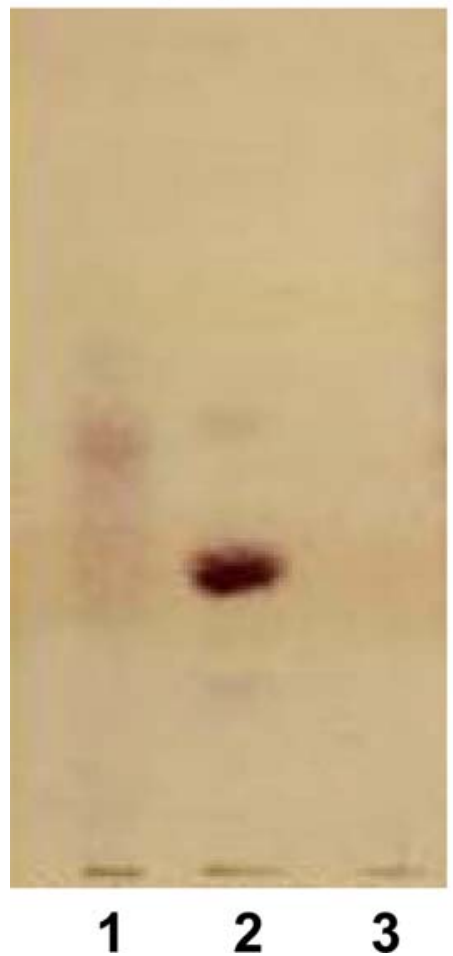

resorcinol

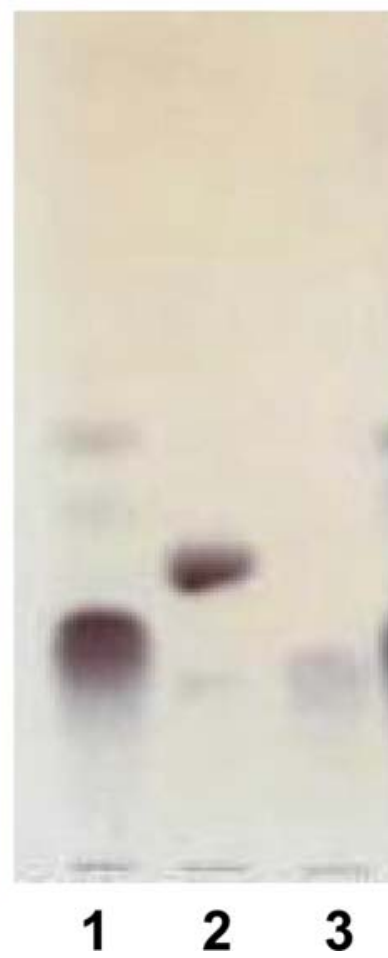

Fig. 3 Thin-layer chromatography (TLC) of the purified material from the patient's urine. Lane 1 purified material from the patient's urine, lane 2 sialyl-lactose, lane $3 \mathrm{~N}$-acetylneuraminic acid. Detection of spots was performed by spraying resorcinol or orcinol reagent

\section{Results}

\section{Enzyme assay}

For differential diagnosis, assaying of the enzymes involved in sialic acid metabolism was performed. The lysosomal sialidase and acid carboxypeptidase activities of cultured fibroblasts from the patient were $46 \mathrm{nmol} / \mathrm{h}$ per milligram of protein [controls, $34 \pm 9$ (10), mean $\pm \mathrm{SD}(n)]$ and $2.1 \mu \mathrm{mol} / \mathrm{h}$ per milligram of protein [controls, $4.0 \pm 1.6(7)$, mean $\pm \mathrm{SD}(n)$ ], respectively.

Biochemical determination of the excreted materials in the patient's urine

Bio-gel P2 column chromatography demonstrated that the major resorcinol-positive band for the patient's urine was eluted as fraction numbers $48-53$, as shown in Fig. 2. A corresponding band was also detected for the control urine, but the content was much lower. The major material was purified with a cellulose cartridge comigrated with authentic $\mathrm{N}$-acetylneuraminic acid (NeuAc) and exhibited resorcinol-positive and orcinolnegative character, as shown in Fig. 3.

The matrix-assisted laser desorption/ionization timeof-flight (MALDI-TOF) mass spectrum demonstrated the presence of molecule-related ions at $m / z$ 332, assigned as $[\mathrm{NeuAc}+\mathrm{Na}]^{+}, m / z 354,[\mathrm{NeuAc}+2 \mathrm{Na}-\mathrm{H}]^{+}$, and $m / z$ 370, $[\mathrm{NeuAc}+\mathrm{Na}+\mathrm{K}-\mathrm{H}]^{+}$(Fig. 4). These results indicate that the excreted substance in the patient's urine is free $N$-acetylneuraminic acid.

The free $N$-acetylneuraminic acid content in the patient's urine, determined by HPLC, was $758 \mu \mathrm{g} / \mathrm{mg}$ creatinine, which for a normal control is $6 \mu \mathrm{g} / \mathrm{mg}$ creatinine. These results indicate that the substance abnormally excreted into the patient's urine is free $\mathrm{N}$-acetylneuraminic acid, and that the patient excretes it at a concentration of more than a 100 -fold that in control subjects.

Identification and localization of storage materials in the patient's cells

To identify the intracellularly accumulated substances, staining with various types of lectins was performed. As shown in Fig. 5, marked granular fluorescence was observed in cultured fibroblasts from the patient compared
Fig. 4 Matrix-assisted laser desorption/ionization time-offlight (MALDI-TOF) mass spectroscopic spectrum of the purified substance from the patient's urine

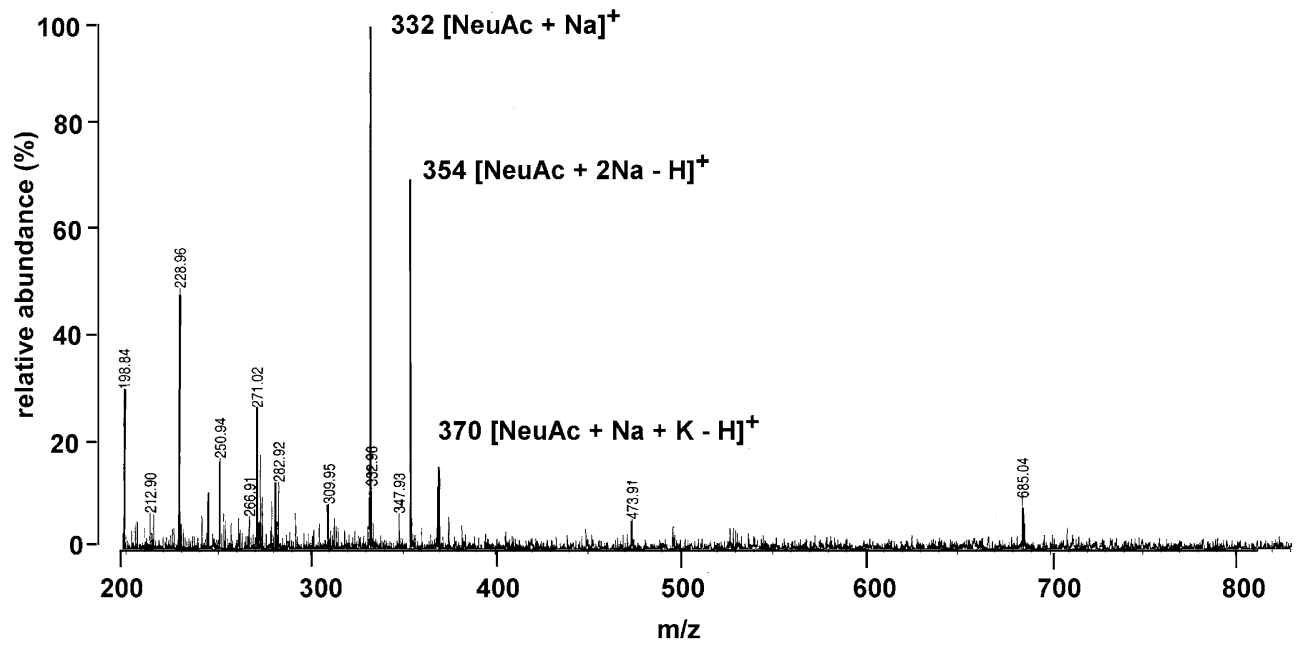


(a)

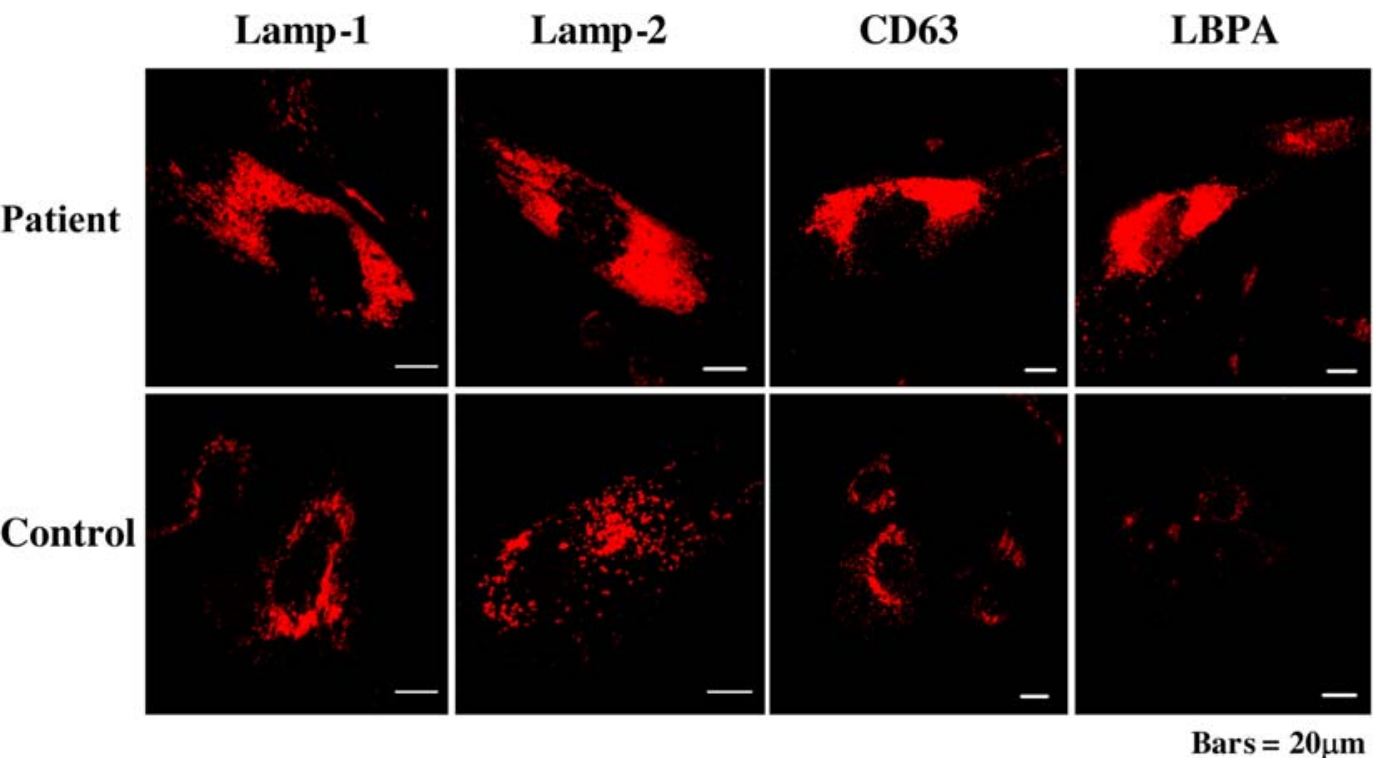

(b)

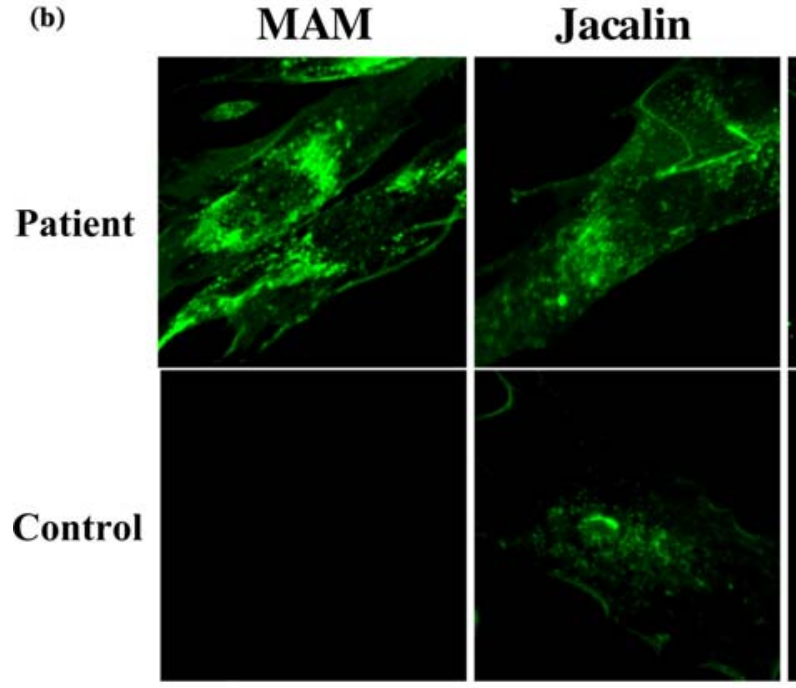

Jacalin

PNA

DSA

Tn
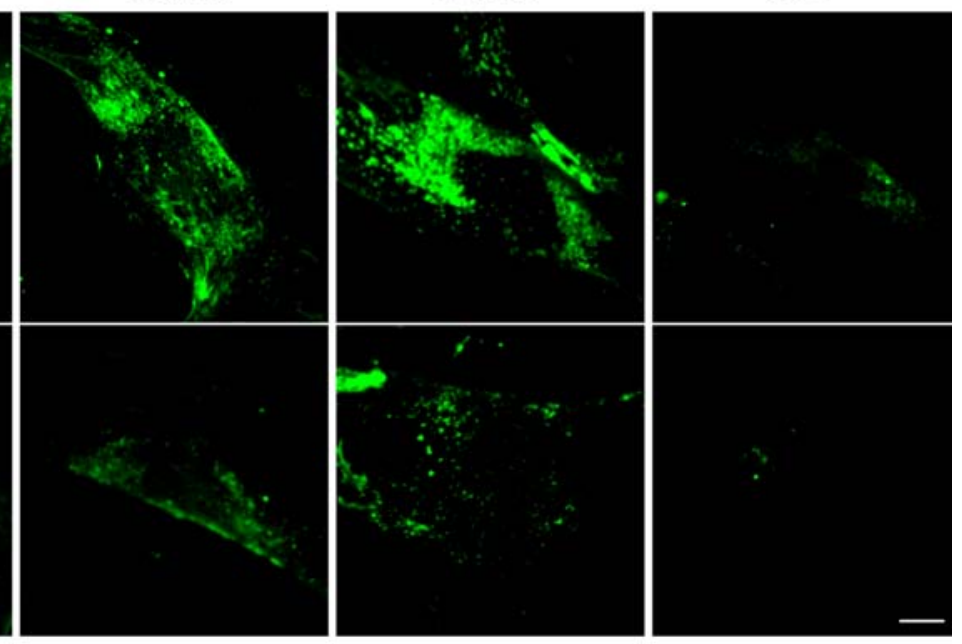

Bar $=20 \mu \mathrm{m}$

Fig. 5 Cytochemical analysis of cultured fibroblasts. a Lamp-1 staining with anti-Lamp-1, Lamp-2 staining with anti-Lamp-2, $C D 63$ staining with anti-CD63, LBPA staining with anti-LBPA. b $M A M$ staining with MAM, Jacalin staining with Jacalin, $P N A$ staining with PNA, DSA staining with DSA, Tn staining with the anti-Tn antigen. Bars $=20 \mu \mathrm{m}$

with a control when the cells were stained with MAM (binding specificity, Sia $\alpha 2-3 \mathrm{Gal}$ ), Jacalin (binding specificity, Sia-Gal $\beta 1-3$ GalNAc- $O-$, Sialyl T-antigen), PNA (binding specificity, Gal $\beta 1-3 \mathrm{GalNAc}>\mathrm{Gal}$ ), and DSA (binding specificity, $N$-acetyl lactosamine). However, no difference in staining pattern was found between cells from the patient and those from controls when the cells were stained with an anti-Tn antigen (binding specificity, GalNAc $\alpha 1-O$-Ser/Thr) (Fig. 5), PHA (binding specificity, GalNAc), and WGA (binding specificity, GlcNAc) (data not shown).

Enhanced granular fluorescence was found in the patient's cells when they were stained with anti-Lamp-1 (a marker of lysosomes), anti-Lamp-2 (a marker of lysosomes), anti-CD63 (a marker of late endosomes), and anti-LBPA (a marker of late endosomes), suggesting that the lesion is located in lysosomes and late endosomes.

Furthermore, we performed lectin blotting with MAM to confirm the presence of MAM-reactive glycoconjugates in the patient's cells. Apparent broad bands in the high molecular region were found for the patient's cells as well for cells from an ISSD patient and sialidosis patients, but these specific bands were not detected for control cells (Fig. 6).

\section{Discussion}

Up to now, only one case (a female) of ISSD in Japan has been reported (Nakano et al. 1996). She presented failure to thrive, coarse facies, hepatosplenomegaly, 


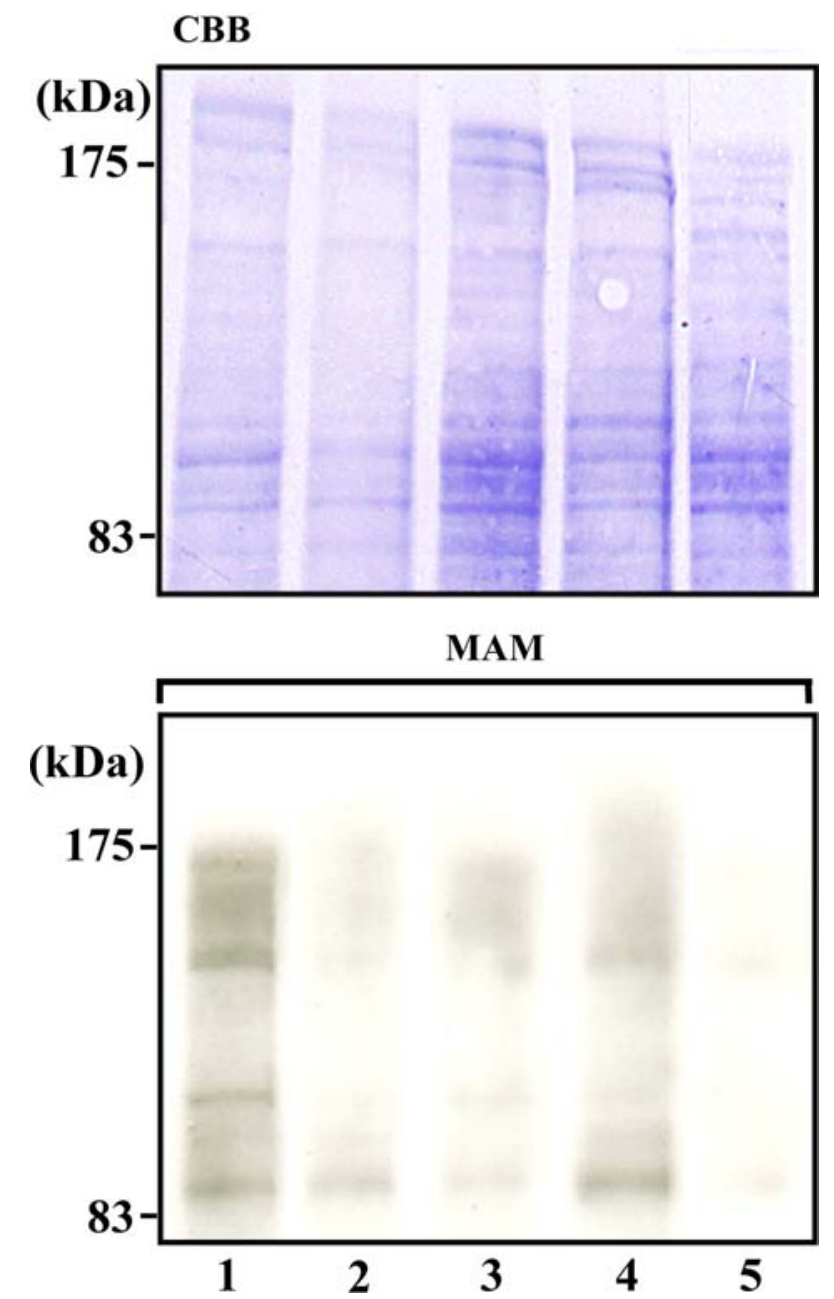

Fig. 6 Lectin blotting of cell lysates from the patient with MAM. $C B B$ staining with Coomassie brilliant blue, $M A M$ staining with Maakia amurensis. Lane 1 the patient, lane 2 a patient with infantile free sialic acid storage disease, lanes 3 and 4 patients with sialidosis, lane 5 a normal subject

severe psychomotor retardation, and spastic quadriplegia. Compared with that case, the clinical manifestations in our patient are mild, and the clinical course is slow. The unique clinical feature of our patient is proteinuria. The association of a renal disorder in lysosomal free sialic acid storage diseases had been thought to be unusual. However, a renal disorder in those diseases has been focused on since Lemyre et al. (1999) reported that a nephrotic syndrome sometimes occurs in ISSD patients. As far as we know, this is the first case that develops renal disorder as the main clinical manifestation in Salla disease. In our case, a renal biopsy, which was performed to determine the cause of the proteinuria, demonstrated prominent vacuolation of glomerular podocytes, mesangial cells, vascular endothelial cells, and proximal tubular epithelial cells. Electron microscopy revealed that hypertrophic podocytes as well as other renal components were filled with numerous membrane-bound vacuoles, which suggests that the main lesion is in lysosomes and late endosomes, and these specific pathological changes must be the cause of the renal disorder in this patient.

In this case, we demonstrated that the major abnormal material excreted into urine is free sialic acid, and not oligosaccharides or glycoproteins, by means of TLC and mass spectrometry. These results well fulfill the diagnostic criteria for Salla disease. The enzyme assay results confirmed that the patient does not have sialidosis (primary lysosomal sialidase deficiency) or galactosialidosis (secondary lysosomal sialidase deficiency due to a primary defect of acid carboxypeptidase) in which a large number of sialyl oligosaccharides are excreted into the urine. Then, we investigated intracellularly accumulated substances with various kinds of lectins that bind to specific sugar moieties. The results showed that both sialyl and asialyl glycoconjugates accumulated in the patient's cells. These results basically coincide with those of Virtanen et al. (1980) who showed that cultured Salladisease fibroblasts reacted with Limulus polyphemus agglutinin, which associates with sialyl glycoconjugates. They thought that Salla disease is related to defective metabolism of sialyl glycoconjugates. The reason sialyl and asialyl glycoconjugates accumulate in lysosomes and late endosomes in this disease is unknown. Sialyl glycoconjugates are degraded within lysosomes through sequential hydrolysis of the terminal sugar moiety, which is usually initiated by lysosomal sialidase. The removed sialic acid residues are usually transported through sialin, a lysosomal membrane protein involved in the transport system for sialic acid, glucuronic acid, and some other acidic monosaccharides (Havelaar et al. 1998), which is encoded by the SLC17A5 gene (Verheijen et al. 1999).

In ISSD and Salla disease, a defect in sialin activity has been shown (Mancini et al. 1991). So, a large amount of free sialic acid residues must be accumulated in lysosomes, and this might secondarily cause a defect in the catalytic metabolism of sialyl glycoconjugates in lysosomes.

We performed gene analysis in this patient according to the method described previously (Verheijen et al. 1999; Aula et al. 2000). Total RNA was isolated from the patient's cultured fibroblasts, and then sialin cDNA was amplified by polymerase chain reaction using the RNA as a template. We sequenced the cDNA, but we could not detect any gene mutation in the protein coding region (data not shown). Totally, 95\% of the Finnish patients with Salla disease have a p.R39C missense mutation in both alleles, the rest of them being compound heterozygous for this mutation (Aula and Gahl 2001). Our patient does not have this common mutation. The disease-causing mutation could be somewhere other than in the protein coding region. Unfortunately, we could not obtain any available antisialin antibodies and could not perform protein analyses for sialin.

In conclusion, we found the first Japanese patient with Salla disease and performed clinical, biochemical, and cytochemical studies on him. The patient developed unique proteinuria besides the characteristic manifestations of Salla disease. A renal disorder should be considered not 
only in ISSD but also in Salla disease. We showed that sialyl and asialyl glycoconjugates accumulated in cultured fibroblasts derived from the patient. Some impairment of sialyl glycoconjugate metabolism occurs due to the defect in the lysosomal free sialic acid transport in this disease.

Acknowledgements The authors would like to thank Professor Kousaku Ohno of the Department of Child Neurology, Tottori University School of Medicine, for proffering cultured fibroblasts from a patient with infantile free sialic acid storage disease. This work was partly supported by grants from the Tokyo Metropolitan Government, The Japan Society for the Promotion of Science, CREST, the Japan Science and Technology Agency, the Ministry of Education, Science, Sports and Culture of Japan, and the Ministry of Health, Labor and Welfare of Japan.

\section{References}

Aula OP, Gahl WA (2001) Disorders of free sialic acid storage. In: Scriver CR, Beaudet AL, Sly WS, Walle D (eds) The metabolic and molecular bases of inherited disease, 8th edn. McGrawHill, New York, pp 5109-5120

Aula N, Salmaki P, Timonen R, Verheijen F, Mancini G, Månsson J-E, Aula P, Peltonen L (2000) The spectrum of SLC17A5-gene mutations resulting in free sialic acid-storage diseases indicates some genotype-phenotype correlation. Am J Hum Genet 67:832-840

Hara S, Yamaguchi M, Takemori Y, Furuhata K, Ogura H, Nakamura M (1989) Determination of mono- $O$-acetylated $\mathrm{N}$ acetylneuraminic acids in human and rat sera by fluorometric high-performance liquid chromatography. Anal Biochem 179:162-166
Havelaar AC, Mancini GM, Beerens CE, Souren RM, Verheijen FW (1998) Purification of the lysosomal sialic acid transporter. Functional characteristics of a monocarboxylate transporter. J Biol Chem 273:34568-34573

Itoh K, Miharu N, Ohama K, Mizoguchi M, Sakura N, Sakuraba H (1997) Fetal diagnosis of galactosialidosis (protective protein/cathepsin A deficiency). Clin Chim Acta 266:72-82

Kobayashi T, Beuchat M-H, Lindsay M, Frias S, Palmiter RD, Sakuraba H, Parton RG, Gruenberg J (1999) Late endosomal membranes rich in lysobisphosphatidic acid regulate cholesterol transport. Nat Cell Biol 1:113-118

Kotani M, Yamada H, Sakuraba H (2004) Cytochemical and biochemical detection of intracellularly accumulated sialyl glycoconjugates in sialidosis and galactosialidosis fibroblasts with Maackia amurensis. Clin Chim Acta 344:131-135

Lemyre E, Russo P, Melancon SB, Gagne R, Potier M, Lambert M (1999) Clinical spectrum of infantile free sialic acid storage disease. Am J Med Genet 82:385-391

Mancini GMS, Beerens CEMT, Aula PP, Verheijen FW (1991) Sialic acid storage diseases. A multiple lysosomal transport defect for acidic monosaccharides. J Clin Invest 87:1329-1335

Nakano C, Hirabayashi Y, Ohno K, Yano T, Mito T, Sakurai M (1996) A Japanese case of infantile sialic acid storage disease. Brain Dev 18:153-156

Sperl W, Gruber W, Quatacker J, Monnens L, Thoenes W, Fink FM, Paschke E (1990) Nephrosis in two siblings with infantile sialic acid storage disease. Eur J Pediatr 149:477-482

Verheijen FW, Verbeek E, Aula N, Beerens CEMT, Havelaar AC, Joosse M, Peltonen L, Aula P, Galjaard H, van der Spek P, Mancini GMS (1999) A new gene, encoding an anion transporter, is mutated in sialic acid storage diseases. Nat Genet 23:462-465

Virtanen I, Ekblom P, Laurila P, Nordling S, Raivio KO, Aula P (1980) Characterization of storage material in cultured fibroblasts by specific lectin binding in lysosomal storage diseases. Pediatr Res 14:1199-1203 